\title{
Preferência lateral e coordenação motora
}

\author{
Lateral preference and motor coordination
}

\author{
Cidália Freitas,,$^{1,2^{*}}$ Manuel Botelho, ${ }^{1,2}$ Olga Vasconcelos ${ }^{1,2}$
}

ARTIGO ORIGINAL | ORIGINAL ARTICLE

\begin{abstract}
Neste estudo pretendemos verificar o efeito da preferência manual (PM) e podal (PP), do sexo e da idade na coordenação motora (CM) em crianças. Para avaliar a PM aplicou-se a Card-reaching task (Carlier et al. 2006, adaptada de Bishop et al., 1996) e o Questionário de PM de Van Strien (2002). Para avaliar a PP recorreu-se à tarefa de pontapear uma bola (Hart \& Gabbard, 1996). A CM foi avaliada através do Movement Assessment Battery for Children (M-ABC) de Henderson e Sugden (1992) em 319 crianças (7.96 \pm 2.38 anos). Os resultados revelam que destrímanos possuem melhor desempenho na destreza manual (DM) e nas habilidades com bola (HB) com a sua mão preferida (Mp). Os sinistrómanos apresentam melhor desempenho com a sua mão não preferida (MNp) na DM. As crianças com PP esquerda são melhores com o seu pé não preferido (PNp) no equilíbrio estático (EE). As meninas apresentaram melhor DM com a MNp e melhor EE com o PNp. Verificamos melhor desempenho da DM com a Mp e MNp, das HB com a MNp e do EE com o pé preferido (Pp) e PNp nos mais novos. Verificou-se um efeito da interação $\mathrm{PM} \times$ Idade e da $\mathrm{PM} \times$ Idade $\times$ Sexo. Concluímos que a lateralidade, o sexo e a idade apresentam efeitos significativos na CM das crianças.

Palavras-chave: lateralidade, coordenação motora, M-ABC, crianças
\end{abstract}

ABSTRACT

The aim of this study was to evaluate the effect of hand and foot preferences, sex and age on motor coordination (MC) in children. We evaluated the hand preference (HP) through the Card-reaching task (Carlier et al., 2006, adapted from Bishop et al., 1996) and the Dutch Handedness Questionnaire (Van Strien, 2002). Foot preference (FP) was evaluated inviting the subjects to kicking a ball (Hart \& Gabbard, 1996). We applied the Movement Assessment Battery for Children MC test (Henderson \& Sugden, 1992) to 319 children $(7.96 \pm 2.38 \mathrm{y})$. The results show that right handers have better performance on manual dexterity (MD) and ball skills (BS) with their preferred hand (PH). Left-handers perform better with their non-preferred hand (NPH) in MD and have a better static balance (SB) with your non-preferred foot (NPF). Girls had better MD with NPH and better SB with NPF. We observed a better MD performance with $\mathrm{PH}$ and NPH, BS with NPH and SB with the PF and NPF in the younger children. $\mathrm{PH} \times$ Age and $\mathrm{Mp} \times$ Age $\times$ Sex interactions were also verified. It can be concluded that the laterality, sex and age have significant effects on children MC.

Keywords: laterality, motor coordination, $\mathrm{M}-\mathrm{ABC}$, children

Artigo recebido a 16.12.2012; $1^{\text {a }}$ Revisão 13.05.2013; 2 ${ }^{\text {a }}$ Revisão 18.10.2013; Aceite 12.01.2014

${ }^{1}$ Faculdade de Desporto da Universidade do Porto (FADEUP), Portugal

${ }^{2}$ Laboratório de Aprendizagem e Controlo Motor, CIFI2D - Universidade do Porto, Portugal

* Autor correspondente: Faculdade de Desporto da Universidade do Porto, Rua Doutor Plácido da Costa, 91, 4200-450 Porto, Portugal; E-mail: cidaliadefreitas@sapo.pt 


\section{INTRODUÇÃO}

A lateralidade funcional pode ser definida como a preferência por um dos membros ou dos órgãos dos sentidos. A preferência manual (PM) é o índice de preferência lateral mais estudado e é expresso pela preferência de utilização de uma mão em relação à outra. Enquanto uns autores observaram uma associação entre a PM e a proficiência manual (Annett, 2004; Bagi, Kudachi, \& Goudar, 2011; Hausmanm, Kirk, \& Corballis, 2004), outros sugerem que nem sempre a mão com melhor desempenho corresponde à mão preferida (Mp) (Porac \& Coren, 1981). Tal como a PM, a preferência podal (PP) constitui uma expressão da lateralidade humana e reporta-se, neste caso, ao pé mais frequentemente utilizado na execução de tarefas motoras unipodais. Estas podem ser divididas em tarefas estáticas, como o equilíbrio (Eq) num só pé, e dinâmicas, como chutar uma bola. Nas estáticas, o pé preferido $(\mathrm{Pp})$ é normalmente utilizado para efetuar o apoio; nas dinâmicas, o Pp é geralmente usado para executar o movimento e o pé não preferido (PNp) serve de apoio. Tal como se verifica na PM, na PP também se distingue a preferência da proficiência. Por exemplo, tarefas envolvendo força, como carregar num pedal, ou tarefas de precisão, como pontapear uma bola para determinada zona da baliza, são executadas pelo pé mais proficiente, que nem sempre coincide com o Pp.

A proporção de sinistrómanos na população é de aproximadamente 10\% (McManus, 2002), podendo variar entre os 0.6 e os $19.8 \%$ dependendo de fatores culturais e étnicos (Porac, Rees, \& Buller, 1990). Muitas teorias tentaram explicar as taxas desproporcionadas entre destrímanos e sinistrómanos. O modelo genético mais amplamente aceite é o Right Shift (Annett, 1985), o qual refere que a lateralidade é definida por um gene que influencia a tendência que o indivíduo apresenta com relação à própria lateralidade. Outra teoria genética (similar), o Dextral Chance (McManus, 1985) propõe que o genótipo DD produz apenas destrímanos, o genótipo CC produz uma mistura aleatória de
$50 \%$ destrímanos e $50 \%$ de sinistrómanos, e o genótipo heterozigoto DC produz $25 \%$ de sinistrómanos e $75 \%$ de destrímanos. Se por um lado, todas as teorias concordam que a PM é biologicamente determinada (Annett, 1985), por outro as teorias envolvem também a aceitação dos constrangimentos sociais e culturais, que conduzem a percentagens diferentes de sinistrómanos entre as várias culturas. A hipótese do mundo orientado à direita ("right-based world", Porac \& Coren, 1981) revela que o ambiente físico favorece os destrímanos e impõe uma adaptação aos sinistrómanos que, face à falta de equipamentos e utensílios apropriados (abre latas, tesouras, ...), são forçados a utilizar a sua mão não preferida ( $\mathrm{MNp}$ ) na realização de tarefas na sua vida diária.

A literatura sugere que os sinistrómanos são menos lateralizados do que os destrímanos (Gurd, Schulz, Cherkas, \& Ebers, 2006) e que na sequência da sua adaptação a um mundo destro são mais proficientes com a sua $\mathrm{MNp}$ (Judge \& Stirling, 2003). Este aspeto tem como consequência uma menor assimetria motora funcional, por parte dos sinistrómanos, a qual parece proporcionar vantagem em tarefas de coordenação combinada de ambos os membros (Gorynia \& Egenter, 2000).

O desenvolvimento de competências motoras depende de vários fatores, como a lateralidade, o sexo e a idade. No âmbito da lateralidade e das assimetrias funcionais dela decorrentes, a literatura revela que os sinistrómanos não são tão lateralizados quanto os destrímanos (Bagi, Kudachi, \& Goudar, 2011; Steenhuis \& Bryden, 1999) e apresentam, comparativamente a estes, uma performance superior com a MNp, em muitas tarefas, como por exemplo, as que envolvem destreza manual (DM) fina ("Peg-moving", Annett \& Kilshaw, 1983), DM fina e força de preensão (Bagi et al., 2011; Steenhuis \& Bryden, 1999) e destreza digital ("Finger tapping", Schmidt, Oliveira, Krahe, \& Filgueiras, 2000). O estudo de Vasconcelos (1993), com 253 crianças dos 11 aos 14 anos, revela que os destros apresentaram melhores desempenhos com a $\mathrm{Mp}$, os sinistrómanos 
foram superiores com a MNp e apresentaram, na tarefa de força, melhores desempenhos, com qualquer das mãos, comparativamente aos destrímanos. Por outro lado, algumas investigações apontam uma melhor coordenação motora em destrímanos (Giagazoglu, Potiadou, Angelopoulou, Tsikoulas, \& Tsimaras, 2001; Kastner-Koller, Deimann, \& Bruckner, 2007). Relativamente ao sexo, alguns autores (e.g., Engel-Yeger, Rosenblum, \& Josman, 2010; Livesey, Coleman, \& Piek, 2007) referem, que as meninas apresentam melhor desempenho no Eq e na DM e que os meninos revelam um melhor desempenho nas habilidades com bola (HB). No entanto, outros estudos não verificaram diferenças na coordenação motora (CM) entre sexos (e.g., Shala, 2009; Venetsanou, \& Kambas, 2010). Quanto à idade, a literatura revela resultados controversos na $\mathrm{CM}$ avaliada através do Movement Assessment Battery for Children (M-ABC) de Henderson e Sugden (1992). Este instrumento é reconhecido a nível mundial como uma medida de ouro na avaliação das dificuldades da CM (Blank, SmitsEngelsman, Polatajko, \& Wilson, 2012). Enquanto alguns estudos verificaram melhores desempenhos por parte das crianças mais velhas (Chow, Yung-Wen, Henderson, Barnett, \& Sing Kai, 2006; Henderson \& Sugden, 1992) outros observaram piores desempenhos (e.g., Silva \& Beltrame, 2013; Valentini et al., 2012).

Como profissionais de Educação Física e Desporto, sobretudo intervindo junto da população infanto-juvenil, devemos ter um conhecimento aprofundado sobre as questões coordenativas, de importância crucial neste período da vida, e da relação destas questões com variáveis que interferem no processo de ensino-aprendizagem. A lateralidade é uma dessas variáveis, pois a literatura remete-nos para um efeito da PM e da PP no desempenho motor de crianças e jovens. São vários os estudos sugerindo, por exemplo, que os sinistrómanos são mais proficientes do que os destrímanos no que respeita à MNp (Judge \& Stirling, 2003) e também que são menos assimétricos do que os destrímanos (Rousson, Gasser, Caflisch, \&
Jenni, 2009). Procurando contribuir para o conhecimento neste domínio, o nosso estudo pretende verificar o efeito da PM, da PP, da idade e do sexo na $\mathrm{CM}$ das crianças, de modo a podermos intervir com mais conhecimento e de forma mais consciente no processo de ensino-aprendizagem.

\section{MÉTODO}

O presente estudo é de natureza experimental e transversal.

\section{Participantes}

Tendo em consideração que o objetivo deste estudo foi comparar as crianças com diferente preferência manual, numa fase inicial realizamos o levantamento de todas as crianças com preferência manual esquerda através da mão da escrita e sem experiência desportiva. Estes dados iniciais foram levantados em crianças entre os 4 aos 12 anos de idade, em 8 escolas públicas pertencentes ao distrito do Porto (Portugal), num total de 1700 crianças. Estas crianças foram convidadas a participar neste estudo e foram selecionadas, posteriormente, de forma aleatória, crianças com a mesma idade, sexo e sem experiência desportiva dentro da mesma turma. Assim, foi elaborada uma amostra de conveniência constituída por 319 crianças, dos 4 aos 12 anos de idade (7.96 \pm 2.38 anos). Os dados relativos à prática desportiva das crianças foram obtidos através de um questionário preenchido pelos encarregados de educação e apenas foram incluídas no estudo as crianças que não apresentavam experiência desportiva extracurricular. A PM foi confirmada através do Card-reaching task (Carlier et al. 2006, adaptada de Bishop et al., 1996) e do Questionário de PM de Van Strien (2002). Assim, 154 crianças foram classificadas como fortemente destrímanas e 119 crianças como fortemente sinistrómanas, tendo sido expurgadas 46 crianças na análise da PM. Relativamente à $\mathrm{PP}$, avaliada através da tarefa de pontapear uma bola (Hart \& Gabbard, 1996), 225 crianças apresentaram PP direita e 94 crianças a PP esquerda. Relativamente ao sexo, 
167 eram do sexo masculino e 151 do sexo feminino. A amostra foi subdividida em 4 grupos de idade, conforme as bandas de idade do teste M-ABC (Henderson \& Sugden, 1992): Banda 1 (4-6 anos, $\mathrm{n}=100)$; Banda 2 (7-8 anos, $\mathrm{n}=89$ ); Banda 3 (9-10 anos, $\mathrm{n}=71$ ) e Banda 4 (11-12 anos, $\mathrm{n}=59)$.

Este estudo foi realizado segundo as normas da Declaração de Helsínquia. Foi obtido um termo de consentimento informado dos encarregados de educação das crianças participantes, no qual foram apresentados os procedimentos, a duração do experimento e os seus direitos como participantes da pesquisa. Os procedimentos experimentais foram aprovados pelo Comité de Ética da Universidade do Porto. Não foram incluídas crianças com necessidades educativas especiais comprovadas ou com diagnóstico de deficiência motora, problemas neurológicos, comportamentais ou ortopédicos.

Todos os participantes realizaram os testes numa sala ampla e isolada, no seu estabelecimento de ensino, durante o tempo letivo. A aplicação dos testes teve uma duração total de aproximadamente 35 minutos.

\section{Instrumentos}

Para avaliar a PM das crianças aplicamos o Card-reaching task (Carlier et al. 2006, adaptada de Bishop et al., 1996) e o Questionário de PM de Van Strien (2002). Para avaliar a PP aplicou-se a tarefa de pontapear uma bola (Hart \& Gabbard, 1996).

Card-reaching task (Carlier et al. 2006, adaptada de Bishop et al., 1996)

Numa mesa são colocados 21 cartões com diferentes figuras, cada uma repetida em 3 cartões sobrepostos, posicionados conforme a figura 1. O participante sentado em frente a uma mesma com as mãos nos joelhos, coloca os cartões no recipiente (caixa), colocado na linha média, conforme a ordem indicada pelo avaliador. É registada a mão utilizada em cada uma das 21 tentativas. A criança é classificada como destrímana quando alcança no mínimo
11 vezes com a mão direita e como sinistrómana quando alcança as cartas no mínimo 11 vezes com a mão esquerda.

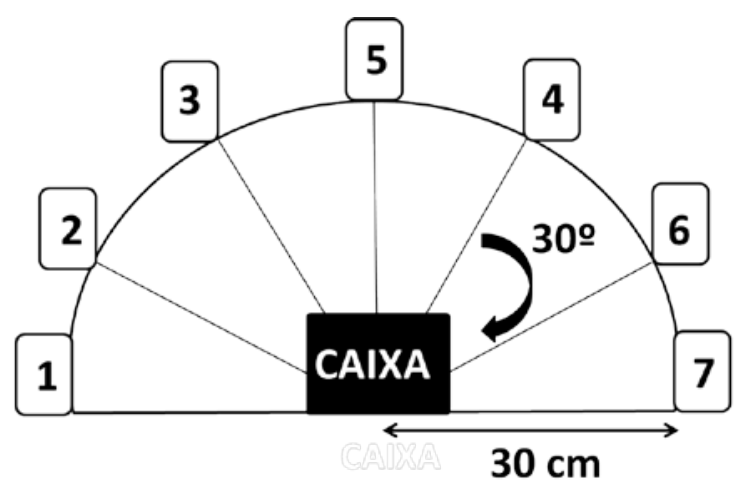

Figura 1. Card-reaching task

Questionário de PM de Van Strien (2002)

Foi registada a mão utilizada em 10 tarefas: pegar no lápis quando desenha; segurar a escova; lavar os dentes; desenroscar a tampa de uma garrafa; lançar uma bola; dar as cartas de um baralho; pegar numa raquete; abrir a tampa de uma caixa; pegar numa colher quando come sopa; apagar com uma borracha; abrir uma porta com uma chave. Para a opção pela mão direita atribuiu-se o valor +1 , para a opção pela mão esquerda o valor -1 , e à opção por "qualquer delas", o valor 0 . Os participantes foram classificados como fortemente destrímanos (com valores entre 8 e 10) e fortemente sinistrómanos (com valores entre -10 e -8 ).

\section{Tarefa de pontapear uma bola (Hart \& Gabbard, 1996)}

Foram classificados com PP direita as crianças que em três tentativas de pontapear uma bola, utilizaram o pé direito pelo menos 2 vezes e com PP esquerda as que utilizaram o pé esquerdo pelo menos 2 vezes.

A CM foi avaliada através do teste motor do M-ABC de Henderson e Sugden (1992). Este teste foi concebido para avaliar os níveis de coordenação motora de crianças dos 4 aos 12 anos de idade, combinando dados quantitativos e qualitativos resultantes da avaliação de testes estandardizados para a motricidade fina e global. Esta bateria é constituída por trinta e duas 
provas e uma lista de verificação que avalia o impacto das dificuldades motoras da criança em contexto educacional. Para cada uma das quatro faixas etárias (4-6 anos; 7-8 anos; 9-10 anos e 11-12 anos), a bateria avalia três capacidades através da execução de oito provas: três de $\mathrm{DM}$, duas de HB, uma de equilíbrio estático (EE) e duas de equilíbrio dinâmico. Esta bateria foi traduzida e adaptada culturalmente para a população portuguesa relativamente às quatro bandas de idade e foram estudadas as suas qualidades através de uma análise da equivalência métrica (sensibilidade e fiabilidade) com amostras da população portuguesa (Cardoso, Silva, Silva, \& Vasconcelos, 2009, 2009). O primeiro passo é registar a pontuação do desempenho de cada tarefa do teste e colocar um "F" se a criança não completar a tarefa, um "I" se a tarefa for inapropriada ou um "R" se a criança não quiser cooperar. Relativamente aos valores de desempenho de cada tarefa este é convertido numa escala de pontuação que varia de zero a cinco, representando as pontuações mais baixas os melhores desempenhos. Assim, o valor zero é atribuído ao sucesso completo numa determinada tarefa avaliada, sendo que o valor cinco significa uma falha. Um "F", "I" ou " $R$ " é convertido numa pontuação de cinco valores.

Para este estudo consideramos apenas as provas motoras unilaterais, isto é, aquelas que são executadas, à vez, pelo membro preferido e pelo membro não preferido. Foi então possível obter, nas 4 bandas de idade, a média da DM (provas 1 e 3) e das HB (prova 5) para a Mp e para a MNp e a média do EE (prova 6) para o Pp e para o PNp (ver Tabela 1). De referir que, nas provas motoras, as crianças foram contrabalançadas em relação ao membro de início da tarefa. A pontuação atribuída, quer para o membro preferido, quer para o membro não preferido, teve por base a mesma cotação, isto é, a do membro preferido.

Tabela 1

Provas do teste $M-A B C$ para as quatro bandas de idade

\begin{tabular}{|c|c|c|c|c|c|}
\hline \multicolumn{2}{|c|}{ Provas } & $\begin{array}{c}\text { Banda } 1 \\
(4-6 \text { anos) }\end{array}$ & $\begin{array}{c}\text { Banda } 2 \\
\text { (7-8 anos) }\end{array}$ & $\begin{array}{c}\text { Banda } 3 \\
\text { (9-10 anos) }\end{array}$ & $\begin{array}{c}\text { Banda } 4 \\
\text { (11-12 anos) }\end{array}$ \\
\hline \multirow{3}{*}{$\mathrm{DM}$} & 1 & $\begin{array}{l}\text { Colocar moedas } \\
\text { num mealheiro }\end{array}$ & Colocar pinos & $\begin{array}{c}\text { Colocar pinos em } \\
\text { linhas }\end{array}$ & $\begin{array}{l}\text { Fixar peças de } \\
\text { madeira }\end{array}$ \\
\hline & 2 & $\begin{array}{l}\text { Enfiar contas num } \\
\text { cordão }\end{array}$ & Enfiar cordão & $\begin{array}{c}\text { Enroscar porcas no } \\
\text { parafuso }\end{array}$ & Recortar elefante \\
\hline & 3 & $\begin{array}{c}\text { Delinear percurso } \\
\text { de bicicleta }\end{array}$ & Delinear flor & Delinear flor & Delinear flor \\
\hline \multirow{2}{*}{$\mathrm{HB}$} & 4 & $\begin{array}{c}\text { Agarrar saco de } \\
\text { feijõos }\end{array}$ & $\begin{array}{l}\text { Arremessar a bola } \\
\text { no chão agarrar } \\
\text { com uma mão }\end{array}$ & $\begin{array}{l}\text { Lançar e agarrar a } \\
\text { bola com duas } \\
\text { mãos }\end{array}$ & $\begin{array}{l}\text { Lançar e agarrar } \\
\text { bola com uma mão }\end{array}$ \\
\hline & 5 & $\begin{array}{c}\text { Rolar bola para a } \\
\text { baliza }\end{array}$ & $\begin{array}{l}\text { Atirar saco de fei- } \\
\text { jões para dentro de } \\
\text { uma caixa }\end{array}$ & $\begin{array}{l}\text { Atirar saco de fei- } \\
\text { jões para dentro de } \\
\text { uma caixa }\end{array}$ & Tiro ao alvo \\
\hline \multirow{3}{*}{$\mathrm{Eq}$} & 6 & $\begin{array}{c}\text { Equilibrar-se sobre } \\
\text { um pé }\end{array}$ & $\begin{array}{l}\text { Equilíbrio da cego- } \\
\text { nha }\end{array}$ & $\begin{array}{c}\text { Equilibrar numa } \\
\text { tábua }\end{array}$ & $\begin{array}{c}\text { Equilibrar em duas } \\
\text { tábuas }\end{array}$ \\
\hline & 7 & $\begin{array}{l}\text { Saltar por cima da } \\
\text { corda }\end{array}$ & $\begin{array}{l}\text { Saltar em quadra- } \\
\text { dos }\end{array}$ & $\begin{array}{l}\text { Saltar ao pé- } \\
\text { coxinho nos } \\
\text { quadrados }\end{array}$ & $\begin{array}{l}\text { Bater palmas e } \\
\text { saltar }\end{array}$ \\
\hline & 8 & $\begin{array}{l}\text { Caminhar em pon- } \\
\text { tas }\end{array}$ & $\begin{array}{l}\text { Caminhar em cal- } \\
\text { canhar-pontas }\end{array}$ & $\begin{array}{l}\text { Equilibrar a bola } \\
\text { em deslocamento }\end{array}$ & $\begin{array}{c}\text { Deslocar à reta- } \\
\text { guarda }\end{array}$ \\
\hline
\end{tabular}




\section{Análise Estatística}

Foi efetuada a análise exploratória dos dados de forma a avaliar a normalidade da distribuição correspondente a cada uma das variáveis em estudo e a eventual presença de outliers, utilizando o teste KolmogorovSmirnov (K-S). Efetuámos uma ANOVA multivariada $2(\mathrm{PM}$ ou $\mathrm{PP}) \times 2$ (sexo) $\times 4$ (banda de idade) para examinar o efeito dos fatores principais no desempenho da CM. O teste post hoc utilizado foi o de Bonferroni e o nível de significância foi fixado em $p \leq 0.05$. Os resultados serão apresentados versando os fatores principais ou interações com significado estatístico.

\section{RESULTADOS}

A Tabela 2 apresenta os valores médios correspondentes às provas que avaliam as capacidades de DM, HB e EE, com os membros preferido e não preferido, para cada grupo de preferência (manual ou podal).

Relativamente à $\mathrm{Mp}$, as crianças fortemente destrímanas apresentam melhores desempenhos do que as crianças fortemente sinistrómanas na DM $\left(F_{1,257}=30.378, p<0.001\right)$ e nas $\mathrm{HB}\left(F_{1,257}=5.481, p=0.020\right)$. Em contraparti$\mathrm{da}$, no que respeita à $\mathrm{MNp}$, as crianças fortemente sinistrómanas apresentam um desempenho significativamente superior na DM $\left(F_{1,257}=20.558, \quad p<0.001\right)$ relativamente às fortemente destrímanas. No que respeita à PP, considerando o PNp, as crianças com PP esquerda demonstram um EE significativamente $\left(F_{1,302}=4.695, p=0.031\right)$ mais proficiente comparativamente às crianças com PP direita. Não se verificaram diferenças significativas entre a preferência direita e esquerda nas HB com a MNp e no EE com o Pp.

A Tabela 3 mostra os valores médios correspondentes às provas que avaliam as capacidades de DM, HB e EE, com os membros preferido e não preferido, para cada sexo.

O sexo feminino obteve um melhor desempenho comparativamente ao sexo masculino, com diferenças estatisticamente significativas na DM com a MNp $\left(F_{1,257}=5.574, p=0.019\right) \mathrm{e}$ no EE com o PNp $\left(F_{1,302}=5.134, p=0.024\right)$. Não se verificaram diferenças significativas entre os sexos nas HB com a Mp e com a MNp, na DM com a Mp e no EE com o Pp.

A Tabela 4 expõe os valores médios relativos às provas que avaliam as capacidades de $\mathrm{DM}, \mathrm{HB}$ e $\mathrm{EE}$, com os membros preferido e não preferido, para as quatro bandas de idade.

Verificamos que a banda de idade 1 (4-6 anos) apresenta um desempenho significativamente superior na DM com a $\mathrm{Mp}\left(F_{3,257}=\right.$ $65.85, p<0.001)$ e com a MNp $\left(F_{3,257}=77.80\right.$, $p<0.001$ ), para a sua idade, comparativamente aos restantes grupos, o mesmo constatamos nas HB com a $\mathrm{MNp}\left(F_{3,257}=4.07, p=0.008\right)$. Observamos que a banda de idade 2 é aquela que apresenta um melhor EE quer com o Pp

Tabela 2

$D M, H B$ e EE com os membros preferido e não preferido para cada grupo de preferência (manual ou podal). Média, desvio padrão, valores de Fe p.

\begin{tabular}{ccccc}
\hline Preferência manual & $\begin{array}{c}\text { Fortemente } \\
\text { Direita }(\mathrm{n}=164)\end{array}$ & $\begin{array}{c}\text { Fortemente } \\
\text { Esquerda }(\mathrm{n}=155)\end{array}$ & $F$ & $p$ \\
\hline DM-Mp & $1.00 \pm 1.15$ & $1.48 \pm 1.36$ & 30.378 & 0.001 \\
DM-MNp & $2.81 \pm 1.57$ & $2.12 \pm 1.45$ & 20.558 & 0.001 \\
HB-Mp & $0.69 \pm 1.27$ & $1.03 \pm 1.42$ & 5.481 & 0.020 \\
HB-MNp & $1.78 \pm 1.65$ & $1.42 \pm 1.53$ & 3.243 & 0.073 \\
\hline Preferência podal & Direita $(\mathrm{n}=225)$ & Esquerda $(\mathrm{n}=94)$ & $F$ & $p$ \\
\hline EE-Pp & $1.67 \pm 1.97$ & $1.37 \pm 1.91$ & 1.870 & 0.173 \\
EE-PNp & $1.91 \pm 2.03$ & $1.48 \pm 1.84$ & 4.695 & 0.031 \\
\hline
\end{tabular}


Tabela 3

DM, HB e EE com os membros preferido e não preferido para cada sexo. Média, desvio padrão, valores de F e p.

\begin{tabular}{ccccc}
\hline & $\begin{array}{c}\text { Masculino } \\
(\mathrm{n}=167)\end{array}$ & $\begin{array}{c}\text { Feminino } \\
(\mathrm{n}=152)\end{array}$ & $F$ & $p$ \\
\hline DM-Mp & $1.33 \pm 1.28$ & $1.08 \pm 1.25$ & 3.527 & 0.062 \\
DM-MNp & $2.60 \pm 1.53$ & $2.40 \pm 1.58$ & 5.574 & 0.019 \\
HB-Mp & $0.73 \pm 1.31$ & $0.96 \pm 1.38$ & 1.442 & 0.231 \\
HB-MNp & $1.55 \pm 1.63$ & $1.71 \pm 1.38$ & 0.091 & 0.763 \\
EE-Pp & $1.69 \pm 1.97$ & $1.47 \pm 1.94$ & 3.659 & 0.057 \\
EE-PNp & $1.87 \pm 1.94$ & $1.68 \pm 2.02$ & 5.134 & 0.024 \\
\hline
\end{tabular}

Tabela 4

$D M, H B$ e EE com os membros preferido e não preferido para cada banda de idade do M-ABC. Média, desvio padrão, valores de Fep.

\begin{tabular}{|c|c|c|c|c|c|c|}
\hline & $\begin{array}{c}\text { Banda } 1 \\
(\mathrm{n}=100)\end{array}$ & $\begin{array}{l}\text { Banda } 2 \\
(n=89)\end{array}$ & $\begin{array}{c}\text { Banda } 3 \\
(n=71)\end{array}$ & $\begin{array}{c}\text { Banda } 4 \\
(n=59)\end{array}$ & $F$ & $p$ \\
\hline DM-Mp & $0.32 \pm 0.60$ & $1.06 \pm .77$ & $2.34 \pm 1.47$ & $1.50 \pm 1.20$ & 65.85 & 0.000 \\
\hline DM-MNp & $1.19 \pm 1.24$ & $2,25 \pm .61$ & $3.86 \pm 1.04$ & $3.36 \pm 1.60$ & 77.80 & 0.000 \\
\hline HB-Mp & $0.61 \pm 1.12$ & $1.03 \pm 1.33$ & $0.83 \pm 1.63$ & $0.96 \pm 1.30$ & 1.41 & 0.239 \\
\hline HB- MNp & $1.34 \pm 1.43$ & $1.59 \pm 1.61$ & $1.48 \pm 1.76$ & $2.31 \pm 1.54$ & 4.07 & 0.008 \\
\hline EE-Pp & $0.79 \pm 1.30$ & $0.61 \pm 1.25$ & $2.62 \pm 1.96$ & $3.15 \pm 2.16$ & 38.82 & 0.000 \\
\hline EE- PNp & $1.09 \pm 1.53$ & $0.78 \pm 1.24$ & $2.90 \pm 1.88$ & $3.10 \pm 2.26$ & 28.59 & 0.000 \\
\hline
\end{tabular}

$\left(F_{3,302}=38.82, p<0.001\right)$, quer com o PNp $\left(F_{3,302}=28.59, p<0.001\right)$, seguindo-se a banda 1 (4-6 anos), a banda 3 (9-10 anos) e, por fim, a banda 4 (11-12 anos). Verificamos um pior desempenho da DM, das HB e do EE nas idades mais avançadas, realçando todavia o carácter transversal e não longitudinal do presente estudo.

Verificamos uma interação estatisticamente significativa $\left(F_{3,257}=6.760, p<0.001\right)$ entre a PM e a Idade, na DM com a Mp. Pela análise da Figura 2, podemos constatar que a diferença encontrada entre fortemente destrímanos e fortemente sinistrómanos é superior nas bandas de idade mais avançadas, com os destrímanos a evidenciar melhor desempenho.

A interação $\mathrm{PM} \times$ Idade $\times$ Sexo também evidenciou um efeito significativo na DM com a $\operatorname{Mp}\left(F_{3,257}=3.290, p=0.021\right)$. Pela análise da Figura 3 e da Figura 4, observamos diferenças superiores entre fortemente sinistrómanos e fortemente destrímanos nas idades mais avançadas (banda de idade 3, dos 9-10 anos, e banda de idade 4, dos 11-12 anos), no sexo masculino e no sexo feminino. Todavia, esta diferença expressa-se diferentemente em cada sexo.

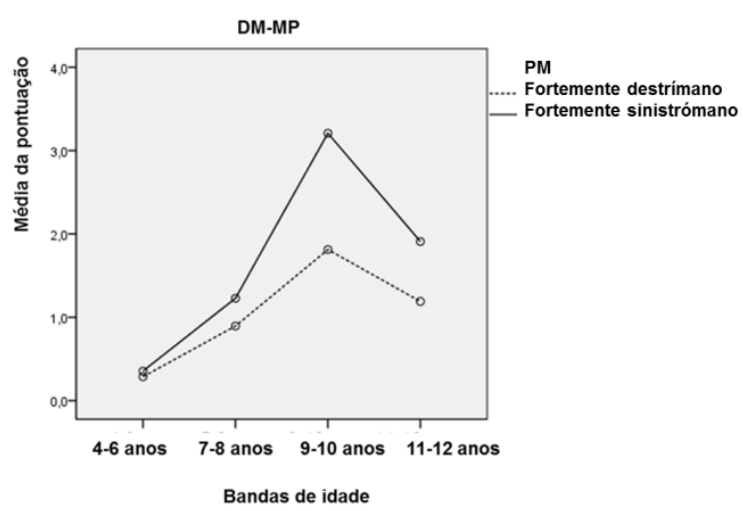

Figura 2. DM com a Mp em fortemente destrímanos e fortemente sinistrómanos, nas 4 bandas de idade 
Verificamos que a DM com a Mp dos meninos destrímanos e sinistrómanos (Figura 3) é semelhante no grupo etário mais novo (4-6 anos) e que através das faixas etárias a diferença entre os dois grupos de PM aumenta. A partir dos 7-8 anos é evidente que os rapazes fortemente destrímanos apresentam uma melhor proficiência com a sua $\mathrm{Mp}$ na DM, comparativamente aos rapazes fortemente sinistrómanos do mesmo grupo de idade. Esta diferença acentua-se nas restantes bandas de idade (9-10 e 11-12 anos).

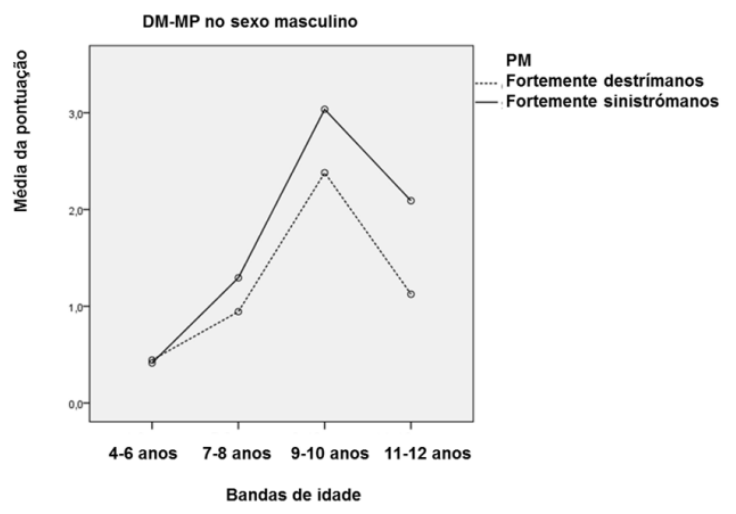

Figura 3. Sexo masculino. DM com a Mp em fortemente destrímanos e fortemente sinistrómanos, nas 4 bandas de idade

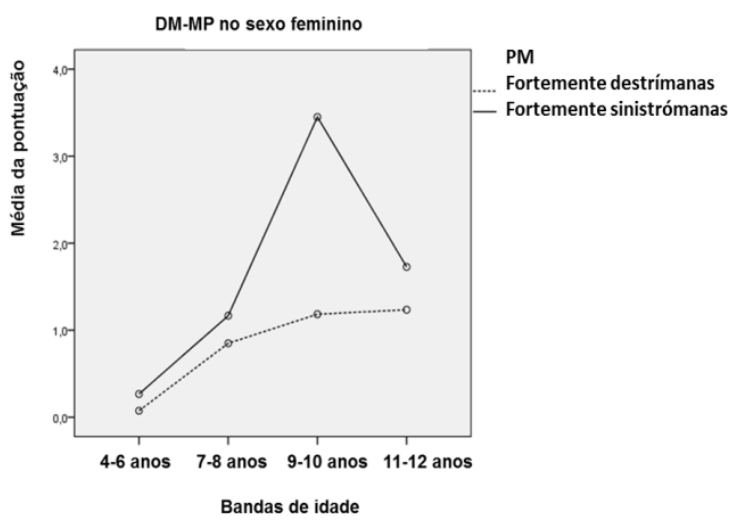

Figura 4. Sexo feminino. DM com a Mp em fortemente destrímanas e fortemente sinistrómanas, nas 4 bandas de idade

Nas meninas fortemente destrímanas e fortemente sinistrómanas, observamos que a DM com a Mp é distinta nas quatro bandas de idade (Figura 4), apresentando-se mais acentuada esta diferença na banda dos 9-10 anos.

\section{DISCUSSÃO}

Com este estudo pretendemos verificar o efeito da PM, da PP, da idade e do sexo na CM de crianças entre os 4 e os 12 anos.

\section{Lateralidade}

Os resultados revelaram, quanto à $\mathrm{PM}$, que as crianças fortemente destrímanas demonstraram um melhor desempenho na DM e nas $\mathrm{HB}$ com a sua $\mathrm{Mp}$, comparativamente às crianças fortemente sinistrómanas. Por sua vez, as crianças fortemente sinistrómanas apresentaram um melhor desempenho com a sua $\mathrm{MNp}$ na DM. Relativamente à $\mathrm{PP}$, as crianças com PP esquerda demonstraram um melhor EE com o seu $\mathrm{PNp}$, comparativamente às crianças com PP direita. Ao observar um efeito da PM na DM, o nosso estudo confirma os resultados de estudos anteriores (Hill \& Khanem, 2009; Vasconcelos, 1993). Embora os autores tenham aplicado outros instrumentos de avaliação da DM, estes verificaram uma melhor proficiência das crianças destrímanas comparativamente às sinistrómanas, ao nível da $\mathrm{Mp}$. $\mathrm{O}$ mesmo se confirmou no que respeita à $\mathrm{MNp}$ na $\mathrm{DM}$, em que os sinistrómanos demonstram melhor performance que os destrímanos. Baseados na ideia suportada por Steenhuis e Bryden (1999), que verificaram que os sinistrómanos não são tão lateralizados quanto os destrímanos, tais resultados sugerem que, apresentando os sinistrómanos uma menor assimetria motora funcional, estes poderão apresentar um melhor desempenho com a sua MNp. Esta hipótese, partilhada mais tarde por Sainburg (2002), denomina-se hipótese dinâmica da dominância na lateralização motora e foi recentemente confirmada em participantes sinistrómanos (Przybyla, Good, \& Sainburg, 2012). Os resultados deste estudo demonstraram que os sinistrómanos, nas tarefas motoras, apresentam um padrão de controlo neuromuscular dos movimentos semelhante ao dos destrímanos. Todavia, no que respeita ao membro não preferido, os sinistrómanos desenvolveram um controlo dos movimentos mais coordenado do que os destrímanos, resultando numa assi- 
metria menos acentuada na sua coordenação intersegmentar, apesar de manterem a prevalência da Mp sobre a MNP, na manifestação da preferência, como têm demonstrado estudos como os de Rodrigues, Lamboglia, Cabral, Barreiros e Vasconcelos (2009). Apesar da menor assimetria, não só na manifestação da preferência mas também na expressão da proficiência, também os sinistrómanos têm apresentado um desempenho superior da sua $\mathrm{Mp}$ no desempenho de tarefas de CM (Gurd et al., 2006). Como foi já referido, esta menor lateralização dos sinistrómanos poderá resultar de uma menor intensidade na preferência pelo uso da Mp como resultado de uma maior frequência no uso da $\mathrm{MNp}$, consequência da vivência num mundo favorecendo o uso do lado direito ("right-based world", Porac \& Coren, 1981). Por outro lado, concordamos com Swinnen, Jardin e Meulenbroek (1996), que justificam os melhores desempenhos dos destrímanos com a sua Mp referindo o facto de estes usarem quase sempre esta mão nas atividades unimanuais e raramente recorrerem à sua MNp. Contrariamente, os sinistrómanos, devido ao facto de viveram num mundo "enviesado" à direita, utilizam com alguma frequência a sua $\mathrm{MNp}$, sendo então esta mais proficiente do que a MNp dos destrímanos. Em consequência, verifica-se uma menor assimetria funcional nos sinistrómanos e alguma perda de funcionalidade da sua Mp. Isto é, os sinistrómanos são "menos sinistrómanos" dos que os destrímanos são destrímanos.

\section{Sexo}

Quanto ao fator sexo, verificaram-se diferenças na DM com a MNp e no EE com o PNp, apresentando o sexo feminino um melhor desempenho do que o sexo masculino. $O$ melhor desempenho na DM por parte do sexo feminino corrobora com vários estudos (Brito \& Santos-Morales, 2002; Ruiz \& Graupera, 2003; Sigmundsson \& Rostoft, 2003; VedulKjelsås, Stensdotter, \& Sigmundsson, 2012), tal como o Eq (Chow et al., 2006; Lam, Ip, Lui, \& Koong, 2003; Lejarraga et al., 2002; Nolan,
Grigorenko, \& Thorstensson, 2005). No entanto, alguns estudos não verificaram diferenças entre sexos na DM (Vedul-Kjelsås et al., 2012) e no equilíbrio (Kourtessis et al., 2008; Shala, 2009; Van Waelvelde, Peersman, Lenoir, Smits Engelsman, \& Henderson, 2008; Venetsanou \& Kambas, 2010). Tal como alguns autores, não encontramos diferenças significativas entre os sexos nas HB (Sigmundsson \& Rostoft, 2003). Contrariamente aos nossos resultados, diversas investigações verificaram um melhor desempenho nas HB por parte dos rapazes com diferenças significativas (Chow et al., 2006; Engel-Yeger et al., 2010; Livesey et al., 2007; Ruiz \& Graupera, 2003; Vedul-Kjelsås et al., 2012). As questões de âmbito cultural foram, em geral, apontadas como justificação para os resultados encontrados nestas investigações. Relativamente ao nosso estudo, como possível explicação, sugerimos que a falta de espaços recreativos e desportivos apropriados para a realização de jogos com bola dos meninos estudados na nossa amostra poderá justificar as semelhanças encontradas nas $\mathrm{HB}$ entre os sexos.

No caso do nosso estudo, pensamos que as diferenças entre os sexos relativas ao membro não preferido, nos testes de DM e de EE, poderão ter origem no envolvimento sociocultural onde a criança se insere. No geral, as raparigas tendem a ser mais estimuladas para tarefas que requerem DM, nomeadamente, para a realização de tarefas que requerem DM fina, por exemplo, a elaboração de colares, a reprodução e recorte de desenhos projetados em papel, fazer penteados (como tranças), bordados e enfeites. Por outro lado, elas tendem a realizar mais jogos recreativos ou tradicionais que apelam ao desenvolvimento do Eq (estático e dinâmico), nomeadamente jogos como o macaquinho chinês, a estátua, a macaca, saltar à corda, entre outros. A DM e o EE podem ser desenvolvidos em atividades executadas com ambos os membros ou apenas com um, podendo ser mais evidente a diferença entre o sexo masculino e feminino com o membro não preferido mais estimulado pelas meninas. 
Por outro lado, os nossos resultados podem ser complementados pelos de Roeder et al. (2008) que verificaram uma menor assimetria funcional nas meninas relativamente aos meninos. Neste estudo foi aplicado o PANESS (Physical Neurological Assessment of Subtle Signs - Exame físico e neurológico de sinais subtis) a 130 crianças destrímanas (65 meninos e 65 meninas), dos 7 aos 14 anos, que realizaram tarefas cronometradas com o pé, mão e dedo. Os resultados revelaram um efeito do sexo, revelando os meninos uma maior diferença entre o lado direito e esquerdo. Os autores justificaram os resultados referindo-se a uma maturação mais precoce, nas meninas, das estruturas cerebrais que suportam a $\mathrm{CM}$ e a velocidade.

\section{Idade}

No que respeita à idade constatamos que o grupo de crianças mais novas (banda 1) apresenta, para a sua idade, um melhor desempenho na DM com a Mp e com a MNp. O mesmo verificou-se nas $\mathrm{HB}$ com a MNp. A banda de idade 2 apresentou um melhor EE, para a sua idade, com o Pp e com o PNp, seguindo-se as bandas 3 e 4 . As idades mais avançadas apresentaram piores resultados, para a sua idade, na DM, nas HB e no EE. Estes resultados corroboram com algumas investigações neste âmbito realizadas com o M-ABC, em que as crianças mais velhas apresentaram piores desempenhos na coordenação motora (EngelYeger et al., 2010; Ruiz \& Graupera, 2003; Silva \& Beltrame, 2013; Valentini et al., 2012). Contrariamente, outros estudos com o M-ABC verificaram um melhor desempenho por parte das crianças mais velhas (Chow et al., 2006; Henderson \& Sugden, 1992; Livesey et al., 2007b). Uma possível interpretação para os nossos resultados, no que respeita à $\mathrm{DM}$, baseia-se na observação das brincadeiras e atividades lúdicas das crianças da nossa amostra, despendendo as crianças mais novas maiores períodos de tempo em atividades que solicitam a DM, as HB e o EE. Além disso, estas experimentam também uma maior diversidade de ações motoras envolvendo as referidas capacidades. Estas atividades, de âmbito recreativo e contextualizadas também no período de tempo destinado à Educação Física, são bastante desenvolvidas na idade pré-escolar. Nesta fase, as crianças possuem muito tempo para as brincadeiras e jogos, nomeadamente com bola, e os professores dispõem de períodos mais alargados de tempo para as estimular e envolver de forma mais intensa nas aprendizagens motoras, com o correspondente desenvolvimento dos padrões básicos do movimento e das respetivas capacidades motoras. Por outro lado, ao entrarem no $1^{\circ}$ Ciclo ou deste para o $2^{\circ}$ Ciclo, as crianças acabam por direcionar essencialmente o seu tempo para as tarefas cognitivas, como a aprendizagem e o desenvolvimento da leitura, da interpretação de textos, da escrita e do cálculo, possuindo menos tempo para se envolver em atividades lúdicas. Outro aspeto que nos parece importante referir na interpretação das diferenças encontradas entre a banda 1 e as outras bandas, é o facto de termos constatado que nas Escolas do Agrupamento da nossa amostra os alunos apenas começam a ter a disciplina de Educação Física a partir do $4^{\circ}$ ano (isto é, a partir dos 9-10 anos de idade). Por outro lado, nas turmas do $1^{\circ}$ Ciclo às quais foi aplicado o M-ABC, a expressão físicomotora não é desenvolvida pela maioria dos professores do $1^{\circ}$ Ciclo, evocando argumentos tais como o extenso conteúdo curricular nas outras áreas (Português, Matemática e Estudo do Meio), a falta de habilitação e de motivação para lecionar esta componente e, principalmente, a limitação, inadequação ou inexistência de espaços recreativos e desportivos, assim como falta de materiais lúdicos e desportivos. Todo este contexto ambiental e educativo parece justificar os resultados encontrados, levando a que os mais novos apresentem, para a sua idade, melhores desempenhos na DM do que os mais velhos. Hirtz e Hultz (1987) referem que é entre os 6 e os 9 anos que as crianças se encontram mais disponíveis para a aquisição e desenvolvimento das capacidades coordenativas, uma vez que nesta fase acontece uma rápi- 
da maturação do sistema nervoso central. Assim, as crianças da nossa amostra parecem não possuir uma estimulação suficiente em idades cujo desenvolvimento da CM é fundamental. Apenas por volta dos 10 anos é que estas crianças voltam a ser estimuladas na escola no sentido de desenvolver os padrões motores básicos e aprender algumas habilidades motoras, que deveriam ter sido desenvolvidos e aprendidas mais cedo. Assim, quando chegam aos 12 anos, elas não apresentam a eficiência que normalmente deveriam demonstrar a nível coordenativo, caso tivesse ocorrido uma estimulação frequente e diversificada ao longo do $1^{\circ}$ Ciclo de ensino. Mais uma vez sugerimos que tal ocorrência poderá explicar o motivo pelo qual as crianças mais velhas apresentaram, para a sua idade, um pior desempenho motor comparativamente às mais novas. Por outro lado, a banda de idade 2 foi aquela que apresentou melhor $\mathrm{EE}$, comparativamente aos restantes grupos de idade. Este resultado pode-se justificar pelo desenvolvimento maturacional da criança de 7-8 anos comparativamente à criança de 4-6 anos e pela escassa estimulação desta capacidade nas restantes bandas de idade subsequentes (9-10 anos e 1112 anos).

\section{CONCLUSÕES}

Concluímos que a PM, o sexo e a idade possuem efeitos significativos na $\mathrm{CM}$ das crianças entre os 4 e os 12 anos de idade. Verificamos que as crianças fortemente destrímanas demonstraram um melhor desempenho na DM e nas HB com a sua Mp e as crianças fortemente sinistrómanas apresentam um melhor desempenho com a sua MNp na DM. As crianças com PP esquerda apresentam um melhor EE com o seu PNp do que as crianças com PP direita. O sexo feminino apresentou melhor DM com a MNp e melhor EE com o PNp do que o sexo masculino. Observamos ainda um melhor desempenho da DM, das HB e do EE nas crianças pertencentes às bandas de idade mais novas. Sugerimos mais investigações neste âmbito englobando a comparação de sujeitos fortemente lateralizados versus sujeitos fracamente lateralizados, à esquerda e à direita, de modo a compreender melhor a relação entre a PM e a CM. Por outro lado, sugerimos mais estudos no sentido de clarificar o efeito da idade e do tipo de envolvimento e de estimulação motora nos vários parâmetros da $\mathrm{CM}$, em crianças do $1^{\circ}$ e $2^{\circ}$ Ciclos de ensino. Independentemente da preferência lateral, da idade ou do sexo, pensamos que é essencial proporcionar às crianças diversas experiências motoras desde os primeiros anos de vida, de modo a desenvolver o seu reportório motor, permitindo-lhes desempenhos mais proficientes e aprendizagens motoras mais eficazes e, sobretudo, mais eficientes ao longo da sua vida.

Agradecimentos:

Nada a declarar.

Conflito de Interesses:

Nada a declarar.

Financiamento:

Nada a declarar.

\section{REFERÊNCIAS}

Annett, M. (1985). Left, right hand and brain: The right shift theory. London: Lawrence Erlbaum Associates.

Annett, M. (2004). Hand preference observed in large healthy samples: classification, norms and interpretations of increased non-right handedness by the right shift theory. British Journal of Psychology, 95(3), 339-353.

Annett, M., \& Kilshaw, D. (1983). Right- and lefthand skill II: Estimating the parameters of the distribution of L-R differences in males and females. British Journal of Psychology, 74(Pt 2), 269-283.

Bagi, J., Kudachi, P., \& Goudar, S. (2011). Influence of motor task on handedness. Al Ameen Journal of Medical Sciences, 4(1), 87-91. 
Bishop, D. V., Ross, V., Daniels, M. S., \& Bright, P. (1996). The measurement of hand preference: A validation study comparing three groups of right-handers. British Journal of Psychology, 87 (2), 269-285.

Blank, R., Smits-Engelsman, B., Polatajko, H., \& Wilson, P. (2012). European Academy for Childhood Disability (EACD): Recommendations on the definition, diagnosis and intervention of developmental coordination disorder (long version). Developmental Medicine \& Child Neurology, 54(1), 54-93. doi: 10.1111/j.14698749.2011.04171.x

Brito, G. N., \& Santos-Morales, T. R. (2002). Developmental norms for the Gardner Steadiness Test and the Purdue Pegboard: A study with children of a metropolitan school in Brazil. Brazilian Journal of Medical and Biological Research, 35, 931-949. doi: 10.1590/S0100-879X200200080 0011

Cardoso, J., Silva, A., Silva, M., \& Vasconcelos, O. (2009). Contributo para a validação da bateria de avaliação Movement Assessment Battery for Children para a população Portuguesa. In L. P. Rodrigues, L. Saraiva, J. Barreiros \& O. Vasconcelos (Eds.), Estudos em Desenvolvimento Motor II (pp. 147-155). Viana do Castelo: ESEIPVC.

Carlier, M., Doyen, A. L., \& Lamard, C. (2006). Midline crossing: Developmental trend from 3 to 10 years of age in a preferential Cardreaching task. Brain and Cognition, 61(3), 255261.

Chow, S. M. K., Yung-Wen, H., Henderson, S. E., Barnett, A. L., \& Sing Kai, L. (2006). The Movement ABC: A cross-cultural comparison of preschool children from Hong Kong, Taiwan, and the USA. Adapted Physical Activity Quarterly, 23(1), 31-48.

Engel-Yeger, B., Rosenblum, S., \& Josman, N. (2010). Movement Assessment Battery for Children (M-ABC): Establishing construct validity for Israeli children. Research in Developmental Disabilities, 31(1), 87-96. doi: 10.1016/ j.ridd.2009.08.001

Giagazoglu, P., Potiadou, E., Angelopoulou, N. Tsikoulas, J. \& Tsimaras, V. (2001). Gross and fine motor skills of left-handed preschool children. Perceptual and Motor Skills, 92, 1122-1128. doi: 10.2466/PMS.92.3.1122-1128

Gorynia, I., \& Egenter, D. (2000). Intermanual coordination in relation to handedness, familial sinistrality and lateral preferences. Cortex, 36(1), 1-18. doi: 10.1016/S0010-9452(08)708 32-3

Gurd, J. M., Schulz, J., Cherkas, L., \& Ebers, G. C. (2006). Hand preference and performance in 20 pairs of monozygotic twins with discordant handedness. Cortex, 42(6), 934-945. doi: 10.1016/S0010-9452(08)70438-6

Hart, S., \& Gabbard, C. (1996). Brief communication: Bilateral footedness and task complexity. The International Journal of Neuroscience, 88(1-2), 141-146.

Hausmann, M., Kirk, I. J., \& Corballis, M. C. (2004). Influence of task complexity on manual asymmetries. Cortex, 40(1), 103-110.

Henderson, S. E., \& Sugden, D.A. (1992). Movement assessment battery for children: Manual. Londres: Psychological Corporation.

Hill, E. L., \& Khanem, F. (2009). The development of hand preference in children: The effect of task demands and links with manual dexterity. Brain and Cognition, 71(2), 99-107. doi: 10.1016/j.bandc.2009.04.006

Hirtz, P., \& Hultz, D. (1987). Como aperfeiçoar as capacidades coordenativas: Exemplos concretos. Horizonte, 3(17), 166-171.

Judge, J, \& Stirling, J. (2003). Fine motor skill performance in left- and right-handers: Evidence of an advantage for left-handers. Laterality, 8(4), 297-306. doi: 10.1080/13576500342000022

Kastner-Koller, U., Deimann, P. \& Bruckner, J. (2007). Assessing handedness in pre-schoolers: Construction and initial validation of a hand preference test for 4-6-year-olds. Psychology Science, 49(3), 239-254.

Kourtessis, T., Thomaidou, E., Liveri-Kantere, A., Michalopoulou, M., Kourtessis, K., \& Kioumourtzoglou, E. (2008). Prevalence of developmental coordination disorder among Greek children with learning disabilities. European Psychomotricity Journal, 1 (2), 10-17.

Lam, M .Y., Ip, M. H., Lui, P. K., \& Koong, M. K. (2003). How teachers can assess kindergarten children's motor performance in Hong Kong. Early Child Development and Care, 173(1), 109118. doi: 10.1080/0300443022000022468

Lejarraga, H., Pascucci, M. C., Krupitzky, S., Kelmansky, D., Bianco, A., Martinez, E., ... Cameron, N. (2002). Psychomotor development in Argentinean children aged 0-5 years. Paediatric Perinatal Epidemiology, 16, 47-60. doi: 10.1046/j.1365-3016.2002.00388.x 
Livesey, D., Coleman, R., \& Piek, J. (2007). Performance on the Movement Assessment Battery for Children by Australian 3- to 5-year-old children. Child: Care, Health and Development, 33(6), 713-719. doi: 10.1111/j.1365-2214.20 07.00733.x

McManus, I. C. (1985). Handedness, language dominance and aphasia: A genetic model. Psychological Medicine Monographs Supplement, 8, 1-140.

McManus, I. C. (2002). Right hand, left hand: The origins of asymmetry in brains, bodies, atoms and cultures. Londres: Weidenfeld and Nicolson.

Nolan, L., Grigorenko, A., \& Thorstensson, A. (2005). Balance control: Sex and age differences in 9- to 16-year-olds. Developmental Medicine \& Child Neurology, 47(7), 449-454. doi: 10.1017/S0012162205000873

Porac, C., \& Coren, S. (1981). Lateral preferences and human behavior. New York: Springer.

Porac, C., Rees, L., \& Buller, T. (1990). Switching hands: A place for left hand use in a right hand world. In S. Coren (Ed.), Left handedness: Behavioural implications and abnormalities. Amsterdam: Elsevier Science.

Przybyla, A., Good, D. C., \& Sainburg, R. L. (2012). Dynamic dominance varies with handedness: reduced interlimb asymmetries in left-handers. Experimental Brain Research. Experimentelle Hirnforschung. Experimentation Cerebrale, 216(3), 419-431. doi: 10.1007/s002 21-011-2946-y

Rodrigues, P., Lamboglia, C., Cabral, I., Barreiros, J., \& Vasconcelos, O. (2009). Degree of hand preference in right- and left-handers: Life-span age trends. Poster presented at The International Seminar Challenges to Sport Sciences, Porto, Portugal.

Roeder, M. B., Mahone, E. M., Larson, J. G., Mostofsky, S. H., Cutting, L. E., Goldberg, M. C., \& Denckla, M. B. (2008). Left-right differences on timed motor examination in children. Child Neuropsychology, 14(3), 249-262. doi: 10.1080/09297040701370016

Rousson, V., Gasser, T., Caflisch, J., \& Jenni, O. G. (2009). Neuromotor performance of normally developing left-handed children and adolescents. Human Movement Science, 28(6), 809-817. doi: 10.1016/j.humov.2009.06.001.

Ruiz, M. L. \& Graupera, J. L. (2003). Competência motriz y género entre escolares españoles. Revista Internacional de Medicina y Ciência de la Actividad Física y el Deporte, 3(10), 101-111.

Sainburg, R. L. (2002). Evidence for a dynamicdominance hypothesis of handedness. Experi- mental Brain Research. Experimentelle Hirnforschung. Experimentation Cerebrale, 142(2), 241-258. doi 10.1007/s00221-001-0913-8

Schmidt, S. L., Oliveira, R. M., Krahe, T. E., \& Filgueiras, C. C. (2000). The effects of hand preference and gender on finger tapping performance asymmetry by the use of an infra-red light measurement device. Neuropsychologia, 38(5), 529-534.

Shala, M. (2009). Assessing gross motor skills of Kosovar preschool children. Early Child Development and Care, 179(7), 969-976. doi: 10.1080/ 03004430701667452

Sigmundsson, H., \& Rostoft, M. S. (2003). Motor development: Exploring the motor competence of 4-year-old Norwegian children. Scandinavian Journal of Educational Research, 47(4), 451-459. doi: org/10.1080/00313830308588

Silva, J., \& Beltrame, T. (2013). Indicativo de transtorno do desenvolvimento da coordenação de escolares com idade entre os 7 e os 10 anos. Revista Brasileira de Ciência do Esporte, 35(1), 314. doi: 10.1590/S0101-32892013000100002

Steenhuis, R. E., \& Bryden, M. P. (1999). The relation between hand preference and hand performance: What you get depends on what you measure. Laterality, 4(1), 3-26. doi: 10.1080/ 713754324

Swinnen, S. P., Jardin, K., \& Meulenbroek, R. (1996). Between-limb asynchronies during bimanual coordination: Effects of manual dominance and attentional cueing. Neuropsychologia, 34(12), 1203-1213.

Valentini, N. C., Coutinho, M. T. C., Pansera, S. M., Santos, V. A. P., Vieira, J. L. L., Ramalho, M. H., \& Oliveira, M. A. (2012). Prevalência de déficits motores e desordem coordenativa desenvolvimental em crianças da região Sul do Brasil. Revista Paulista de Pediatria, 30(3), 377384. doi: 10.1590/S0103-05822012000300011

Van Waelvelde, H., Peersman, W., Lenoir, M., Smits Engelsman, B. C., \& Henderson, S. E. (2008). The movement assessment battery for children: Similarities and differences between 4- and 5year-old children from Flanders and the United States. Pediatric Physical Therapy, 20, 30-38. doi: 10.1097/PEP.0b013e31815ee2b2

Vasconcelos, O. (1993). Asymmetries of manual motor response in relation to age, sex, handedness, and occupational activities. Perceptual and Motor Skills, 77(2), 691-700. 
Vedul-Kjelsås, V., Stensdotter, A., \& Sigmundsson, H. (2012). Motor Competence in 11-Year-Old Boys and Girls. Scandinavian Journal of Educational Research, 57(5), 1-10. doi: 10.1080/003138 31.2012.732603
Venetsanou, F., \& Kambas, A. (2010). Environmental factors affecting preschoolers' motor development. Early Childhood Education Journal, 37(4), 319-327. doi: 10.1007/s10643-009-0350-z

Todo o conteúdo da revista Motricidade está licenciado sob a Creative Commons, exceto quando especificado em contrário e nos conteúdos retirados de outras fontes bibliográficas. 